EPJ Web of Conferences 41, 02030 (2013)

DOI: $10.1051 /$ epjconf/20134102030

C) Owned by the authors, published by EDP Sciences, 2013

\title{
Intra-cluster dynamics induced in molecular clusters by femtosecond UV radiation
}

\author{
Sergey V. Chekalin ${ }^{1}$, Victor O. Kompanets ${ }^{1}$, Valentin M. Apatin ${ }^{1}$, Danil D. Ogurok ${ }^{1}$, \\ Valery N. Lokhman ${ }^{1}$, Denis G. Poydashev ${ }^{1,2}$ and Evgeny A. Ryabov ${ }^{1}$ \\ ${ }^{1}$ Institute for Spectroscopy RAS, 142190, Troitsk, Moscow Region, Russia \\ ${ }^{2}$ Moscow Institute of Physics and Technology (MIPT), 141700, Dolgoprudny, Moscow Region, \\ Russia
}

\begin{abstract}
Dynamics of intra-cluster processes induced by femtosecond UV laser radiation in molecular clusters of $\mathrm{CF}_{3} \mathrm{I}$ and $\mathrm{ICF}_{2} \mathrm{COF}$ molecules is studied. The measured time constants of the observed reactions are within the range from $1 \mathrm{ps}$ to dozens of picoseconds.
\end{abstract}

\section{Introduction}

The results of the studies of intra-cluster processes induced in Van der Waals clusters of $\mathrm{CF}_{3} \mathrm{I}$ and $\mathrm{ICF}_{2} \mathrm{COF}$ molecules by femtosecond UV radiation are presented. UV ionization of pulsed molecular/cluster beams combined with time-of-flight mass-spectrometry was used to measure the mass-spectrum of produced ions and its dependence on laser radiation parameters (wavelength $\lambda$, intensity, polarization, and pulse duration).

\section{Experimental}

The measurements were performed at a setup similar to that described in [1]. Briefly, $\left(\mathrm{CF}_{3} \mathrm{I}\right)_{\mathrm{n}}$ and $\left(\mathrm{ICF}_{2} \mathrm{COF}\right)_{\mathrm{n}}$ clusters were generated by means of gas-dynamic cooling of a $(\mathrm{R}-\mathrm{I})_{\mathrm{n}}+\mathrm{Ar}(1: 15)$ mixture in the supersonic flow of the gas through a pulsed nozzle (General Valve, $d=0.8 \mathrm{~mm}$ ). The backing pressure $P_{0}$ over the nozzle was $200 \mathrm{kPa}$. A skimmer (Beam Dynamics, Model 1, $0.49 \mathrm{~mm}$ diameter) located at a distance of $55 \mathrm{~mm}$ from the nozzle was used to cut a molecular/cluster beam.

The beam of particles thus formed arrived at the chamber of a time-of-flight mass spectrometer. The cluster beam intersected at a distance of $157 \mathrm{~mm}$ from the input of the skimmer with the mutually perpendicular axes of the mass spectrometer and the focused $(\mathrm{f}=30 \mathrm{~cm})$ laser beam used for the UV ionization of the objects under study. In this work we use second and third harmonics of Ti:Sa laser. $\lambda=266 \mathrm{~nm}$ with a pulse duration of $160 \mathrm{fs}$ as well as $\lambda=400 \mathrm{~nm}$ radiation of approx. 100 fs duration was used in most experiments.

\section{Results and discussion}

The yield of ion products was measured as a function of laser energy fluence at $\lambda=400 \mathrm{~nm}$ and 266 $\mathrm{nm}\left(\sim 100\right.$ fs pulse duration) for $\left(\mathrm{CF}_{3} \mathrm{I}\right)_{\mathrm{n}}$ clusters. Both mass-spectrum and yield-fluence dependencies

This is an Open Access article distributed under the terms of the Creative Commons Attribution License 2.0, which permits unrestricted use, distribution, and reproduction in any medium, provided the original work is properly cited. 
were found to be quite different from that observed for nanosecond ( $10 \mathrm{~ns})$ [1] irradiation. Besides of $\mathrm{CF}_{3}{ }^{+}, \mathrm{I}_{2}^{+}$and $\mathrm{I}^{+}$, the molecular ion $\mathrm{CF}_{3} \mathrm{I}^{+}$and a number of more heavier fragments (e.g. $\left(\mathrm{CF}_{3} \mathrm{I}\right)_{2} \mathrm{~F}_{2}{ }^{+}$, $\left(\mathrm{CF}_{3} \mathrm{I}\right)_{2} \mathrm{IF}_{3}^{+}$, etc) were observed for femtosecond irradiation.

It was found for femtosecond irradiation that the measured yield-fluence dependencies in the case of cluster beam differ significantly from those for the free molecules. Such dependencies for $\lambda=$ $266 \mathrm{~nm}$ approximated by a power function shows power index $\mathrm{m} \geq 6$ for ions in the case of cluster ionization instead of $\mathrm{m}=2.15-2.55$ in the case of monomer ionization at the same laser energy fluence range (below $\sim 10^{12} \mathrm{Wcm}^{-2}$ ). It follows that the excitation conditions and probably the ionization mechanism of the free molecules and the same molecules incorporated into clusters are different.

\section{$3.1 \mathrm{I}_{2}^{+}$formation kinetics}

The most intriguing feature of $\left(\mathrm{CF}_{3} \mathrm{I}\right)_{\mathrm{n}}$ cluster ionization process is the formation of molecular ion $\mathrm{I}_{2}{ }^{+}$. It was concluded earlier [1] that $\mathrm{I}_{2}^{+}$ion is a result of intra-cluster photochemical reaction and it is also a precursor to $\mathrm{I}^{+}$ion which, in turn, is produced by a single-photon dissociation of $\mathrm{I}_{2}^{+}$, but the time scale and the mechanism of this reaction remained unknown.

We are reporting here for the first time the measured dynamics of intra-cluster processes induced in $\left(\mathrm{CF}_{3} \mathrm{I}\right)_{\mathrm{n}}$ clusters during UV ionization of these particles. The pump-probe technique was used to measure the kinetic curves. The part of the data obtained for $\mathrm{I}_{2}^{+}$and $\mathrm{I}^{+}$ions is presented on Figure 1.

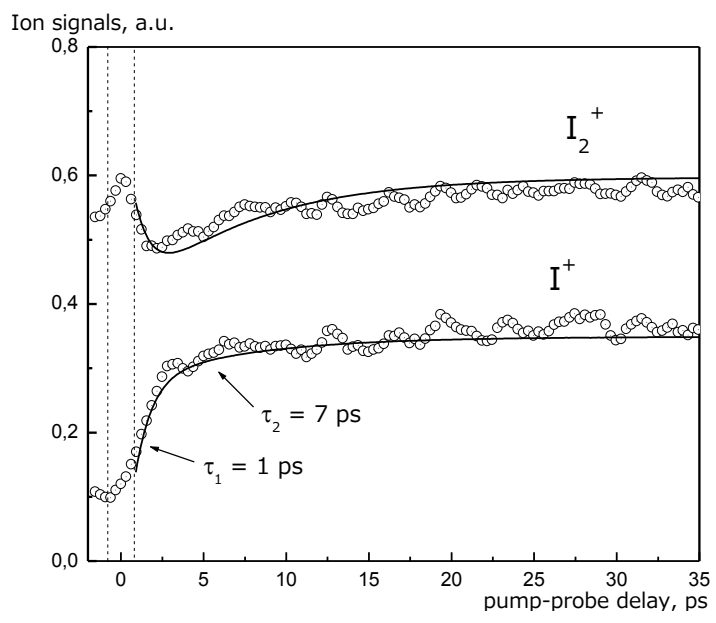

Fig. 1. $\mathrm{I}_{2}{ }^{+}, \mathrm{I}^{+}$ion signals versus pump-probe delay time $\left(\lambda_{\mathrm{PUMP}}=\lambda_{\mathrm{PROBE}}=266 \mathrm{~nm}, \Phi_{\mathrm{PUMP}} \approx 0.1 \mathrm{Jcm}^{-2}\right.$, $\Phi_{\mathrm{PROBE}} \approx 0.05 \mathrm{Jcm}^{-2}$ ).

The measured kinetics of $\mathrm{I}^{+}$signal point to the existence of at least two channels of $\mathrm{I}_{2}^{+}$formation with the time constant of about $\tau_{1} \approx 1$ ps for the fastest one and $\tau_{2} \approx 7$ ps for another one.

Detailed measurements of velocity distribution as well as signal anisotropy were carried out for the ion products under study. Based both on the kinetic and static results obtained the possible mechanism of laser induced intra-cluster reactions is proposed [2].

\subsection{Free neutral molecule formation kinetics}

The reactions induced by the pump pulse at relatively low intensity (below ionization threshold) were observed, too. The frequency of the pump pulse $(\lambda=266 \mathrm{~nm})$ was in resonance with the first 
excited electronic state of the studied $\mathrm{CF}_{3} \mathrm{I}$ and $\mathrm{ICF}_{2} \mathrm{COF}$ molecules. In the case of $\left(\mathrm{CF}_{3} \mathrm{I}\right)_{\mathrm{n}}$ clusters increasing the time interval between pump pulse and ionizing probe one leads to an increase in output of the $\mathrm{CF}_{3} \mathrm{I}^{+}, \mathrm{CF}_{3}{ }^{+}, \mathrm{I}^{+}$ions. It should be noted that the mass-peak shape of the first one looks very much the same as in the case of ionization of the free molecules. This fact gives evidence that cluster excitation by the pump pulse results in production of the neutral free molecules. After that, these molecules are ionized by the probe pulse. Typical rise time of the signal for $\mathrm{CF}_{3} \mathrm{I}^{+}$ion is $\tau_{3} \approx 28$ ps. In the case of irradiation of $\left(\mathrm{ICF}_{2} \mathrm{COF}\right)_{\mathrm{n}}$ clusters the formation of $\mathrm{ICF}_{2} \mathrm{COF}^{+}$molecular ion with a rise time $\tau_{4} \approx 15 \mathrm{ps}$ was observed (data are presented on Figure 2).

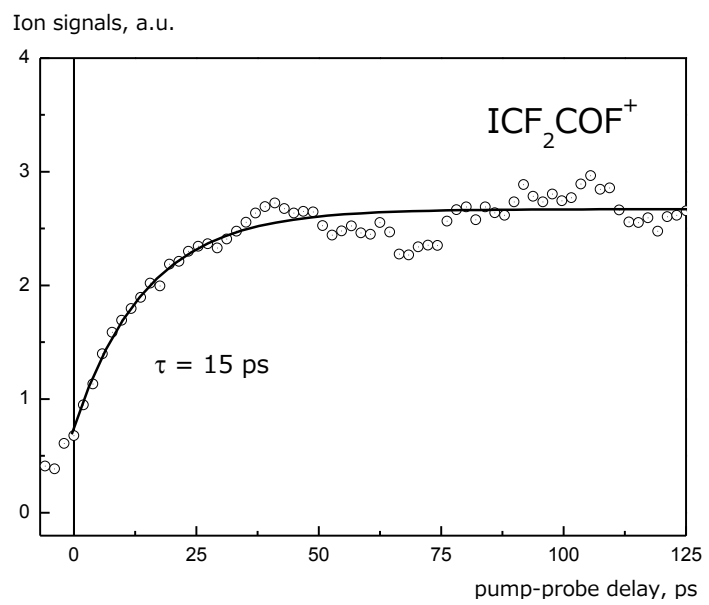

Fig. 2. $\mathrm{ICF}_{2} \mathrm{COF}^{+}$ion signal versus pump-probe delay time $\left(\lambda_{\mathrm{PUMP}}=266 \mathrm{~nm}, \lambda_{\mathrm{PROBE}}=400 \mathrm{~nm}, \Phi_{\mathrm{PUMP}} \approx\right.$ $0.14 \mathrm{Jcm}^{-2}, \Phi_{\mathrm{PROBE}} \approx 0.94 \mathrm{Jcm}^{-2}$ ).

These facts give rise to conclude that the observed kinetics reproduce the emergence of free neutral molecules from the cluster as a result of their electronic excitation. The observed characteristic time thus reproduces the entire set of related processes, including electronic energy relaxation and subsequent cluster decay with formation of free molecule.

It is concluded that with the excitation of electronic states of molecules in the cluster (below the ionization threshold) quite fast conversion of electronic excitation into the internal vibrations of the cluster occurs, followed by its decay and the formation of free molecules. The characteristic time scale of these processes lies in the range of a few dozens of picoseconds.

\section{References}

1. V.M. Apatin, V.N. Lokhman, D.D. Ogurok, D.G. Poydashev and E.A. Ryabov, JETP, 112, 1 (2011)

2. V.M. Apatin, V.O. Kompanets, V.N. Lokhman, N.-D.D. Ogurok, D.G. Poydashev, E.A. Ryabov and S.V. Chekalin, JETP letters, 94, 7 (2011) 